\title{
Multidirectional Power Flow Control among Double Winding Six-Phase Induction Machine Winding Sets
}

\author{
A. A. Abduallah, O. Dordevic, M. Jones \\ Department of Electronics and Electrical Engineering \\ Liverpool John Moores University \\ Liverpool, United Kingdom \\ A.Abduallah@2016.ljmu.ac.uk O.Dordevic@ljmu.ac.uk M.Jones2@1jmu.ac.uk
}

\begin{abstract}
This paper presents an indirect rotor-field oriented control (IRFOC) algorithm for symmetrical six-phase doublewinding induction machines with four three-phase sub-winding sets. The presented algorithm introduces the ability to control the power flow between different sub-winding sets. Multiple threephase induction machines are utilised for critical applications such as more-electric aircrafts, due to their high reliability and fault tolerant capabilities. In this paper, the proposed control scheme is utilising the auxiliary currents of each six-phase sub-motor to achieve full control of the sub-winding sets current amplitudes and consequently the power flow direction for each sub-winding set. A six-phase induction machine with double winding is utilised to validate the proposed control scheme. Four isolated voltage source inverters (VSIs) are used to supply each winding set of the machine. The double-winding six-phase induction machine controlled by the proposed IRFOC algorithm is simulated using Matlab/Simulink. Presented simulation results validate the ability of the algorithm to appropriately control the power flow of each sub-winding set.
\end{abstract}

Keywords - IRFOC; Multidirectional Power Flow; Multiphase Induction Machines; Multiple Three-Phase Machine.

\section{INTRODUCTION}

Recently, multiphase machines with multiple three-phase sub-winding sets started attracting attention of the researchers from the renewable energy area (especially wind energy generation). This is due to the possibility to apply wellestablished three-phase power conversion technology to each of the sub-winding sets $[1,2]$. Moreover, these machines are employed in industrial applications where high level of reliability and fault tolerant capability is compulsory, such as more-electric aircrafts [3-5]. In these applications, the fault tolerance is the main concern. Thus, by increasing the number of phases of the induction machine and having multiple voltage source inverters (VSIs) supplying the machine, fault tolerant capability of the more-electric aircraft increases [3].

One of the recognizable advantages of utilising the multiphase machines is the ability to split the load power among multiple VSI's legs. Additionally, multiphase machines have more degrees of freedom compared to their three-phase counterparts, which are commonly used for fault tolerant applications [3, 6]. Several post-fault strategies have been introduced in the literature relating to a fault occurring in one or more phases of the multiphase machine or in the switches of the VSI $[3,4,7]$. These strategies are commonly using the auxiliary currents to achieve post-fault operation.
As already mentioned, there are several practical applications of the multiple three-phase induction machines such as, more-electric aircrafts [3-5] and electrical vehicles (EVs) [8-11]. In the latter application, the electrical power generation and distribution systems are expected to raise from the conventional low dc voltage to a higher dc voltage level in the near future. This will reduce the system losses and size as well [12]. Furthermore, new starter-alternator topologies with different number of turns per winding set are introduced in [12]. The motivation being to accommodate multiple dc voltage levels (dc-bus voltage level of the corresponding VSIs), present in such system. The introduced starter-alternator is a multiplewinding three-phase induction machine, where each threephase winding represents a sub-motor. The authors used a multiple vector control scheme in order to achieve multidirectional power flow between the multiple three-phase sub-motors. Obviously, multiple three-phase machines can also be categorised as a type of multiphase machines. However, multiple vector control is not the best approach to control multiphase machines. A more popular control method for multiphase machines is based on VSD (vector space decomposition) approach.

High-performance ac electric drives require decoupled control of the flux and torque in a similar way as it is inherently present in the separately excited dc machines [13]. Therefore, in order to apply a fully decoupled control, it is essential to convert the ac machine model into its dc machine equivalent. One variant of this control scheme is indirect rotor-field oriented control (IRFOC), where two fictitious currents allow separate control of the torque and flux of the ac machine [13]. However, due to the non-ideal characteristics of the multiphase machines and power electronics converters in practice, e.g. the dead-time effect and asymmetries of the windings, controlling only the flux and torque producing plane with IRFOC, is not sufficient. Therefore, the other, losses producing planes, should be controlled as well [1, 2, 14, 15].

The aim of this paper is to develop an IRFOC algorithm for a six-phase induction machine with double-windings. The scheme uses the additional degrees of freedom offered by the six-phase machine to control the currents' amplitude and power flow direction of each three-phase sub-winding set.

The paper is organised as follows. Section two gives the mathematical model of the double-winding six-phase induction machine necessary to design the control system. Next, the proposed IRFOC control scheme with the additional ability to 


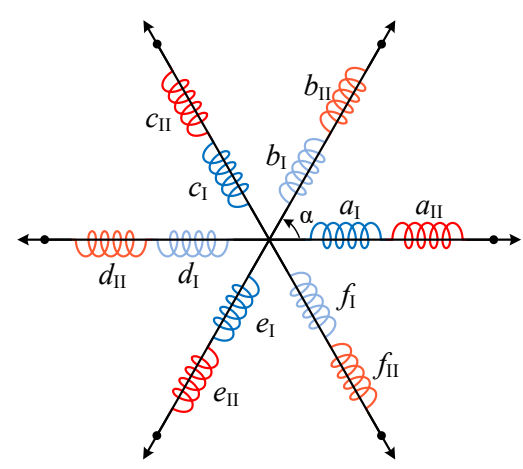

Fig. 1. Spatial position of the symmetrical six-phase double-winding induction motor stator magnetic axes.

control the power flow among the sub-winding sets is derived. In section four, the simulation results of the proposed control strategy are presented. Finally, the concluding remarks are stated in section five.

\section{Double-Winding SiX-Phase Induction Machine Model}

Simplified structure of the double-winding symmetrical sixphase induction machine, illustrating the stator's magnetic axes, is shown in Fig. 1. Mathematical model of this machine can be considered as a twelve-phase machine in phase variable form, but also as a summation of dynamic equations of the two separate six-phase induction sub-motors. This fact makes it possible to simplify control, and is used to control this twelve phase machine as two six-phase sub-motors, as demonstrated in the next section. Also, in order to simplify the equations, the model of the double-winding six-phase induction motor is derived here as a combination of two separate six-phase submotors sharing the same rotor. Stator and rotor voltage equilibrium equations, for each six-phase sub-motor, can be defined as:

$$
\begin{aligned}
& {\left[v_{s k}\right]=\left[R_{s k}\right]\left[i_{s k}\right]+\frac{\mathrm{d}\left[\psi_{s k}\right]}{\mathrm{d} t}} \\
& {\left[v_{r k}\right]=\left[R_{r k}\right]\left[i_{r k}\right]+\frac{\mathrm{d}\left[\psi_{r k}\right]}{\mathrm{d} t}}
\end{aligned}
$$

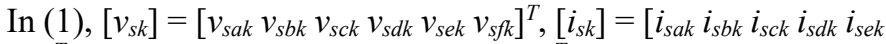
$\left.i_{s f k}\right]^{T},\left[\psi_{s k}\right]=\left[\psi_{s a k} \psi_{s b k} \psi_{s c k} \psi_{s d k} \psi_{s e k} \psi_{s f k}\right]^{T}$, represent stator phase voltages, currents and flux linkages, respectively. Index $k$ is used to indicate sub-motor ( $k=\mathrm{I}$ or II). Matrix $\left[R_{s k}\right]$ represents stator resistance matrix, of each sub-motor. It is a diagonal matrix, where all elements on the main diagonal are equal to $R_{s}$ due to the assumed machine's symmetry. Equation (2) is of the same form as (1), but it is given for rotor variables, hence $r$ in index instead of $s$. Note that division of rotor into two submotors does not physically exist. For instance, if the rotor currents are taken as an example, $\left[i_{r \mathrm{I}}\right]$ and $\left[i_{r \mathrm{II}}\right]$ would represent the rotor currents produced by each sub-motor.

The flux-linkage equations for each sub-motor are defined as follows:

$$
\begin{gathered}
{\left[\psi_{s k}\right]=\left[L_{s k}\right]\left[i_{s k}\right]+\left[L_{s r k}\right]\left[i_{r k}\right]} \\
{\left[\psi_{r k}\right]=\left[L_{r k}\right]\left[i_{r k}\right]+\left[L_{s r k}\right]^{T}\left[i_{s k}\right]}
\end{gathered}
$$

where $\left[L_{s k}\right]$ and $\left[L_{r k}\right]$ represent stator and rotor self-inductance matrices and $\left[L_{s r k}\right]$ represents stator-to-rotor inductance, for $k$-th ( $k=$ I or II) sub-motor. These inductances are defined as (note that below it is assumed that the sub-motors are identical hence the index $k$ is omitted):

$$
\begin{aligned}
& {\left[L_{s}\right]=\left[\begin{array}{cccccc}
L_{l s}+M & \frac{1}{2} M & \frac{-1}{2} M & -M & \frac{-1}{2} M & \frac{1}{2} M \\
\frac{1}{2} M & L_{l s}+M & \frac{1}{2} M & \frac{-1}{2} M & -M & \frac{-1}{2} M \\
\frac{-1}{2} M & \frac{1}{2} M & L_{l s}+M & \frac{1}{2} M & \frac{-1}{2} M & -M \\
-M & \frac{-1}{2} M & \frac{1}{2} M & L_{l s}+M & \frac{1}{2} M & \frac{-1}{2} M \\
\frac{-1}{2} M & -M & \frac{-1}{2} M & \frac{1}{2} M & L_{l s}+M & \frac{1}{2} M \\
\frac{1}{2} M & \frac{-1}{2} M & -M & \frac{-1}{2} M & \frac{1}{2} M & L_{l s}+M
\end{array}\right]} \\
& {\left[L_{r}\right]=\left[\begin{array}{cccccc}
L_{l r}+M & \frac{1}{2} M & \frac{-1}{2} M & -M & \frac{-1}{2} M & \frac{1}{2} M \\
\frac{1}{2} M & L_{l r}+M & \frac{1}{2} M & \frac{-1}{2} M & -M & \frac{-1}{2} M \\
\frac{-1}{2} M & \frac{1}{2} M & L_{l r}+M & \frac{1}{2} M & \frac{-1}{2} M & -M \\
-M & \frac{-1}{2} M & \frac{1}{2} M & L_{l r}+M & \frac{1}{2} M & \frac{-1}{2} M \\
\frac{-1}{2} M & -M & \frac{-1}{2} M & \frac{1}{2} M & L_{l r}+M & \frac{1}{2} M \\
\frac{1}{2} M & \frac{-1}{2} M & -M & \frac{-1}{2} M & \frac{1}{2} M & L_{l r}+M
\end{array}\right]}
\end{aligned}
$$

$\left[L_{s r}\right]=M\left[\begin{array}{cccccc}\cos \theta_{e} & \cos \left(\theta_{e}-\alpha\right) & \cos \left(\theta_{e}-2 \alpha\right) & \cos \left(\theta_{e}-3 \alpha\right) & \cos \left(\theta_{e}-4 \alpha\right) & \cos \left(\theta_{e}-5 \alpha\right) \\ \cos \left(\theta_{e}-5 \alpha\right) & \cos \theta_{e} & \cos \left(\theta_{e}-\alpha\right) & \cos \left(\theta_{e}-2 \alpha\right) & \cos \left(\theta_{e}-3 \alpha\right) & \cos \left(\theta_{e}-4 \alpha\right) \\ \cos \left(\theta_{e}-4 \alpha\right) & \cos \left(\theta_{e}-5 \alpha\right) & \cos \theta_{e} & \cos \left(\theta_{e}-\alpha\right) & \cos \left(\theta_{e}-2 \alpha\right) & \cos \left(\theta_{e}-3 \alpha\right) \\ \cos \left(\theta_{e}-3 \alpha\right) & \cos \left(\theta_{e}-4 \alpha\right) & \cos \left(\theta_{e}-5 \alpha\right) & \cos \theta_{e} & \cos \left(\theta_{e}-\alpha\right) & \cos \left(\theta_{e}-2 \alpha\right) \\ \cos \left(\theta_{e}-2 \alpha\right) & \cos \left(\theta_{e}-3 \alpha\right) & \cos \left(\theta_{e}-4 \alpha\right) & \cos \left(\theta_{e}-5 \alpha\right) & \cos \theta_{e} & \cos \left(\theta_{e}-\alpha\right) \\ \cos \left(\theta_{e}-\alpha\right) & \cos \left(\theta_{e}-2 \alpha\right) & \cos \left(\theta_{e}-3 \alpha\right) & \cos \left(\theta_{e}-4 \alpha\right) & \cos \left(\theta_{e}-5 \alpha\right) & \cos \theta_{e}\end{array}\right]$

In (5)-(7), $M$ represents the maximum value of the mutual inductance between the stator and rotor, while $L_{l s}$ and $L_{l r}$ represent the leakage inductance of the stator and rotor, respectively. In (7), angle $\theta_{e}$ denotes the electrical position of the rotor. Electrical position of the rotor can be determined from the rotor electrical speed, $\omega_{e}$, as:

$$
\theta_{e}=\int \omega_{e} \mathrm{~d} t
$$

The electrical speed of the rotor is related to the mechanical rotor speed through the number of pole pairs $P$ as, $\omega_{e}=P \omega_{m}$. Newton's law for rotation is defined as:

$$
T_{e}-T_{l}=\frac{J}{P} \frac{\mathrm{d} \omega_{e}}{\mathrm{~d} t}
$$

where $T_{e}$ represents the electromagnetic torque, $T_{l}$ is the load torque and $J$ is the inertia of the machine. Finally, the electromagnetic torque $T_{e}$ can be calculated as:

$$
T_{e}=P\left[i_{s}\right]^{T} \frac{\mathrm{d}\left[L_{s r}\right]}{\mathrm{d} \theta_{e}}\left[i_{r}\right]
$$

where $\left[i_{s}\right]$ represents the sum of $\left[i_{s \mathrm{I}}\right]$ and $\left[i_{s \mathrm{II}}\right]$, and $\left[i_{r}\right]$ represents the sum of $\left[i_{r \mathrm{I}}\right]$ and $\left[i_{r \mathrm{II}}\right]$. In other words, $T_{e}$ can be calculated by summing the electrometric torque produced by each six-phase sub-motor. The same approach was applied in [12]. Hence, one can write:

$$
T_{e}=P\left(\left[i_{s \mathrm{I}}\right]^{T} \frac{\mathrm{d}\left[L_{s r \mathrm{I}}\right]}{\mathrm{d} \theta_{e}}\left[i_{r \mathrm{I}}\right]+\left[i_{s \mathrm{II}}\right]^{T} \frac{\mathrm{d}\left[L_{s r \mathrm{II}}\right]}{\mathrm{d} \theta_{e}}\left[i_{r \mathrm{II}}\right]\right)
$$

Note that the parameters of the sub-motors are reliant on the turns ratio between the sub-motors [12]. However, as already mentioned, in this paper it is assumed that two sub-motors, that form the double-winding six-phase machine, are identical. 
In order to implement the IRFOC algorithm, it is essential to convert the ac machine model into its dc machine equivalent where the flux and torque are decoupled. The fictitious flux and torque producing variables can be obtained from the phase variables reference frame through Clarke's decoupling transformation [7]. The Clarke's transformation for a symmetrical six-phase machine with two isolated neutral points, is defined as:

$$
[C]=\sqrt{\frac{2}{6}}\left[\begin{array}{c:cccccc}
\alpha & 1 & \cos (\alpha) & \cos (2 \alpha) & \cos (3 \alpha) & \cos (4 \alpha) & \cos (5 \alpha) \\
\beta & 0 & \sin (\alpha) & \sin (2 \alpha) & \sin (3 \alpha) & \sin (4 \alpha) & \sin (5 \alpha) \\
x & 1 & \cos (2 \alpha) & \cos (4 \alpha) & \cos (6 \alpha) & \cos (8 \alpha) & \cos (10 \alpha) \\
y & 0 & \sin (2 \alpha) & \sin (4 \alpha) & \sin (6 \alpha) & \sin (8 \alpha) & \sin (10 \alpha) \\
0_{+} & 1 & 0 & 1 & 0 & 1 & 0 \\
0 & 0 & 1 & 0 & 1 & 0 & 1
\end{array}\right]
$$

Where $\alpha-\beta$ components are the flux and torque producing variables, while the $x-y$ components are loss-producing components. Components in $x-y$ are usually nullified in order to eliminate the dead-time effect or the other non-linear effects introduced by the power electronics conversion systems or the machine's windings [2]. The major drawback of the decoupled model (after application of the Clarke's transformation only) is presented in the time-dependent coefficients [7], and rotational (Park's) transformation is commonly used to eliminate this problem. The rotational speed of the new introduced common reference frame may be assumed to be arbitrary, $\omega_{a}$. The instantaneous position of the $d$-axis with respect to the first stator winding, $\theta_{s}$, is utilised to transfer the stator's variables of the decoupled model into the rotational ones. The following matrix represents the rotational transformation matrix for the stator's $\alpha-\beta$ components:

$$
\left[D_{s}\right]=d_{s}\left[\begin{array}{cc}
\cos \theta_{s} & \sin \theta_{s} \\
q_{s}-\sin \theta_{s} & \cos \theta_{s}
\end{array}\right]
$$

In (13) the angle $\theta_{s}$, is defined as:

$$
\theta_{s}=\int \omega_{a} \mathrm{~d} t
$$

The rotational transformation of (13) is implemented only on the $\alpha-\beta$ components since the stator-to-rotor coupling terms appear only in this plane. The $\omega_{a}$ can be arbitrarily chosen and for the IRFOC algorithm it is chosen to be equal to the rotational speed of the rotor field [13]. With this choice, the following relations can be obtained:

$$
\theta_{s}=\phi_{r} \quad \omega_{r}=\frac{\mathrm{d} \phi_{r}}{\mathrm{~d} t} \quad \omega_{a}=\omega_{r}
$$

where $\phi_{r}$ and $\omega_{r}$ represent the rotor field instantaneous position and the speed of the rotating field of the rotor, respectively.

\section{CONTROL STRATEGY}

The IRFOC algorithm can be applied to six-phase induction machines utilising previous decoupling and rotational transformations. As already discussed, due to the special construction of the considered double-winding six-phase machine, the control can be implemented by applying IRFOC algorithm to each sub-motor individually [12]. Traditional IRFOC can be reconfigured to suit this specific application. This will yield to a double vector control scheme to control each sub-motor independently. Nevertheless, in order to ensure that the flux of both sub-motors rotates at the same frequency and direction, the $d$-axis current components of the two sub-motors must be synchronised (aligned). The total flux should be maintained constant and divided among sub-motors according to their corresponding nominal flux values. In general, this is determined by the turns ratio, but in this paper sub-motors are assumed to be identical. Furthermore, the $q$-components of the stator currents are proportional to the electromagnetic torque produced by each sub-motor and also must be synchronised.

In IRFOC, in steady state, the $q$-component of the rotor fluxlinkage will be equal to zero. Based on this, the following equation can be found:

$$
\psi_{d r k}=\psi_{r k} \quad \psi_{q r k}=0 \quad \frac{\mathrm{d} \psi_{q r k}}{\mathrm{~d} t}=0
$$

Thus, the reference electromagnetic torque and rotor-flux linkage $\left(T_{e}\right.$ and $\left.\psi_{r}\right)$ can be related to the $i_{d s}$ and $i_{q s}$ as follows:

$$
\begin{gathered}
T_{e}=P\left(L_{m} / L_{r}\right) \psi_{r} i_{q s} \\
T_{e}=P\left(\psi_{r}^{2} / R_{r}\right) \omega_{s l} \\
\psi_{r}=L_{m} i_{d s}
\end{gathered}
$$

where $L_{m}=6 / 2 M, L_{r}=L_{l r}+L_{m}$ and $\omega_{s l}$ represents the slip speed of the induction motor, $\omega_{s l}=\left(\omega_{r}-\omega_{e}\right)$. Based on (16) to (19), the IRFOC scheme for six-phase machine can be implemented as shown in Fig. 2. Since measuring the rotor-flux-linkage position $\phi_{r}$ is difficult, it is normally estimated using the measured speed and estimated parameters of the machine. Based on (15) one can write:

$$
\phi_{r}=\int \omega_{r} \mathrm{~d} t=\int\left(\omega_{e}+\omega_{s l}^{*}\right) \mathrm{d} t=\theta_{e}+\int \omega_{s l}^{*} \mathrm{~d} t
$$

Asterisk is used to denote estimated value, $\omega_{s l}^{*}$. The value of $\omega_{s l}^{*}$ in (20) can be determined based on (17) and (18):

$$
\omega_{s l}^{*}=L_{m} i_{q s}^{*} / T_{r} \psi_{r}^{*}
$$

where $T_{r}$ represents the rotor time constant and it is equal to $\left(L_{r} / R_{r}\right)$. Finally, by using (19), the slip speed can be expressed by using the $d-q$ current components only, as:

$$
\omega_{s l}^{*}=\frac{i_{q s}^{*}}{T_{r} i_{d s}^{*}}=S G \cdot i_{q s}^{*}
$$

where $S G$ stands for the slip gain. For a six-phase induction machine with double sub-motors, the slip speed can be found by adding the influence of $i_{d s}$ and $i_{q s}$ of the two sub-motors as:

$$
\omega_{s l}^{*}=\frac{i_{q s \mathrm{I}}^{*}+\left(N_{s \mathrm{II}} / N_{s \mathrm{I}}\right) \cdot i_{q s \mathrm{II}}^{*}}{\left(\left(L_{m \mathrm{I}}+L_{l r}\right) / R_{r}\right) \cdot\left(i_{d s \mathrm{I}}^{*}+\left(N_{s \mathrm{II}} / N_{s \mathrm{I}}\right) \cdot i_{d s \mathrm{II}}^{*}\right)}
$$

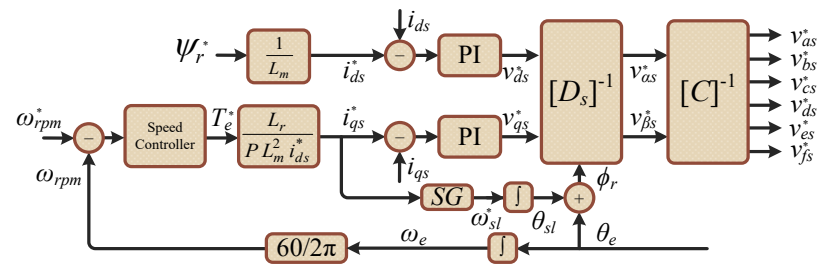

Fig. 2. IRFOC scheme in the synchronous reference frame for six-phase induction machine. 
where $N_{s k}$ indicates the number of turns in each sub-motor. As already mentioned, in this paper the simplest case where $N_{s \mathrm{I}}=$ $N_{s I I}$ is considered. The magnetizing inductance of the first submotor $L_{m \mathrm{I}}$ is equal to $(6 / 2) \cdot M_{\mathrm{I}}$.

In order to utilise the double six-phase machines in starteralternator applications, the IRFOC algorithm for each submotor must share the same speed controller in order to ensure synchronisation of the rotating fields between the sub-motors. However, to obtain multidirectional power flow between them, the speed controller output $\left(i_{q s}\right)$ should be multiplied by a suitable factor to control the current direction of each sub-motor as shown in Fig. 3. Introduced values $G_{\mathrm{I}}$ and $G_{\mathrm{II}}$ are the power sharing gains of two sub-motors. This will allow the power sharing between the sub-motors. However, controlling the power flow direction for each sub-winding set, using these gains only, is not possible. Therefore, further modifications of the proposed IRFOC algorithm and introduction of current sharing coefficients for each sub-winding set are necessary, as explained further.

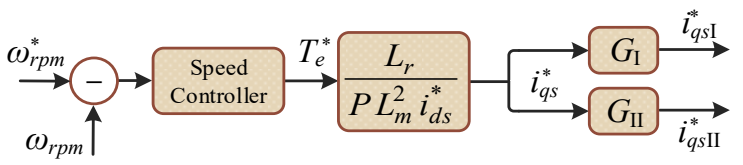

Fig. 3. Modified speed controller for six-phase induction machine with double winding.

As stated before, controlling the $d-q$ currents with IRFOC algorithm is not sufficient. Therefore, the other $x-y$ currents (auxiliary currents) need to be controlled beside the $d-q$ currents. This is common when controlling multiphase machines, and usually it is used to eliminate the non-ideal characteristics of the machine or the power electronics converter. Moreover, post-fault strategies utilise these currents to control the current's amplitude of the winding sets during the post-fault condition. Post-fault control due to a fault of one or more VSIs can be achieved by changing the sharing coefficients of the three-phase winding sets $[2-4,16]$.

In order to control the auxiliary currents in multiple threephase machines, each three-phase sub-winding set can be considered as a three-phase machine. The spatial displacement between each consecutive sub-winding sets in symmetrical induction machines is equal to $\alpha=2 \pi / 6$. Using the decoupling matrix for three-phase induction machines, the contribution of each sub-winding set into the decoupled sub-motor's currents can be obtained. In order to get the decoupled currents of each sub-winding set, the original phase variables currents of each sub-winding set should be multiplied by the three-phase decoupling transformation matrix, taking into consideration the spatial displacement of each winding set. The decoupling matrix of the sub-winding sets can be expressed as:

$$
[C(\delta)]=\sqrt{\frac{2}{3}}\left[\begin{array}{ccc}
\cos (\delta) & \cos (\delta+2 \pi / 3) & \cos (\delta+4 \pi / 3) \\
\sin (\delta) & \sin (\delta+2 \pi / 3) & \sin (\delta+4 \pi / 3) \\
1 / \sqrt{2} & 1 / \sqrt{2} & 1 / \sqrt{2}
\end{array}\right]
$$

where $\delta$ represents the spatial displacement of the sub-winding sets, with respect to the first sub-winding set. For symmetrical six-phase machine, $\delta$ is equal to zero for the first sub-winding set, and $\delta$ is equal to $\delta=\alpha=2 \pi / 6$ for the second sub-winding set. Therefore, (24) can be applied on each sub-winding set as (the equations are given for the first sub-motor $k=\mathrm{I}$, only):

$$
\left[\begin{array}{l}
i_{a \mathrm{I}} \\
i_{c \mathrm{I}} \\
i_{e \mathrm{I}}
\end{array}\right]=[C(0)]^{-1}\left[\begin{array}{l}
i_{\alpha 1} \\
i_{\beta 1} \\
i_{01}
\end{array}\right] \quad\left[\begin{array}{l}
i_{b \mathrm{I}} \\
i_{d \mathrm{I}} \\
i_{f \mathrm{I}}
\end{array}\right]=[C(\alpha)]^{-1}\left[\begin{array}{l}
i_{\alpha 2} \\
i_{\beta 2} \\
i_{02}
\end{array}\right]
$$

The results of calculation from (25) are combined together, defining $\left[i_{p h} \mathrm{I}\right]$ matrix, as:

$$
\left[i_{p h \mathrm{I}}\right]=\left[\begin{array}{c}
i_{a \mathrm{I}} \\
i_{b \mathrm{I}} \\
i_{c \mathrm{I}} \\
i_{d \mathrm{I}} \\
i_{e \mathrm{I}} \\
i_{f \mathrm{I}}
\end{array}\right]=\left[\begin{array}{c}
\frac{\sqrt{2} i_{\alpha 1}}{\sqrt{3}}+\frac{i_{01}}{\sqrt{3}} \\
\frac{i_{\alpha 2}}{\sqrt{6}}+\frac{i_{\beta 2}}{\sqrt{2}}+\frac{i_{02}}{\sqrt{3}} \\
-\frac{i_{\alpha 1}}{\sqrt{6}}+\frac{i_{\beta 1}}{\sqrt{2}}+\frac{i_{01}}{\sqrt{3}} \\
-\frac{\sqrt{2} i_{\alpha 2}}{\sqrt{3}}+\frac{i_{02}}{\sqrt{3}} \\
-\frac{i_{\alpha 1}}{\sqrt{6}}-\frac{i_{\beta 1}}{\sqrt{2}}+\frac{i_{01}}{\sqrt{3}} \\
\frac{i_{\alpha 2}}{\sqrt{6}}-\frac{i_{\beta 2}}{\sqrt{2}}+\frac{i_{02}}{\sqrt{3}}
\end{array}\right]
$$

By multiplying the sub-motor's currents from (26) with the decoupling transformation matrix of the first sub-motor with double neutral points $[C]$ from (12), the product will define how each sub-winding set contributes to the stationary reference frame's currents $\left[i_{\text {Clarke }}\right]$. After calculation one gets:

$$
\left[i_{\text {Clarke }}\right]=\left[\begin{array}{c}
i_{\alpha} \\
i_{\beta} \\
i_{x} \\
i_{y} \\
i_{0+} \\
i_{0-}
\end{array}\right]=[C]\left[i_{p h \mathrm{I}}\right]=\left[\begin{array}{c}
\frac{i_{\alpha 1}+i_{\alpha 2}}{\sqrt{2}} \\
\frac{i_{\beta 1}+i_{\beta 2}}{\sqrt{2}} \\
\frac{i_{\alpha 1}-i_{\alpha 2}}{\sqrt{2}} \\
\frac{-i_{\beta 1}+i_{\beta 2}}{\sqrt{2}} \\
i_{01} \\
i_{02}
\end{array}\right]
$$

From (27), one can see that the stationary currents of the first sub-motor (the same applies for the second sub-motor) consist of $\alpha$ and $\beta$ components of the two sub-winding sets only.

The six-phase sub-motors current space vectors can be defined as:

$$
\begin{gathered}
\bar{i}_{\alpha \beta}=i_{\alpha}+j i_{\beta}=I_{\alpha \beta} e^{j \phi_{\alpha \beta}} \\
\bar{i}_{x y}=i_{x}+j i_{y}=I_{x y} e^{j \phi_{x y}}
\end{gathered}
$$

The sub-winding sets current space vectors can be defined as:

$$
\begin{gathered}
\bar{i}_{\alpha \beta 1}=i_{\alpha 1}+j i_{\beta 1}=I_{\alpha \beta 1} e^{j \phi_{\alpha \beta 1}} \\
\bar{i}_{\alpha \beta 2}=i_{\alpha 2}+j i_{\beta 2}=I_{\alpha \beta 2} e^{j \phi_{\alpha \beta 2}}
\end{gathered}
$$

Now, based on (27) - (29), the sub-motor current space vectors can be expressed in terms of the sub-winding sets current space vectors, as:

$$
\begin{aligned}
& \bar{i}_{\alpha \beta \mathrm{I}}=\frac{1}{\sqrt{2}}\left(\bar{i}_{\alpha \beta 1}+\bar{i}_{\alpha \beta 2}\right) \\
& \bar{i}_{x y \mathrm{I}}=\frac{1}{\sqrt{2}}\left(\bar{i}_{\alpha \beta 1}^{*}-\bar{i}_{\alpha \beta 2}^{*}\right)
\end{aligned}
$$

Note that (30) represents the space vector form (complex notation) of the previously given relationships in (27). 
By introducing the current sharing coefficients $K_{1}, K_{2}, K_{3}$ and $K_{4}$ the sub-winding sets current space vectors can be rewritten in terms of the $\alpha-\beta$ space vectors for each sub-motor. The following relationships can be obtained for the sub-motors:

$$
\begin{aligned}
& \bar{i}_{\alpha \beta 1}=\sqrt{2} K_{1} \bar{i}_{\alpha \beta \mathrm{I}} \\
& \bar{i}_{\alpha \beta 2}=\sqrt{2} K_{2} \bar{i}_{\alpha \beta \mathrm{I}} \\
& \bar{i}_{\alpha \beta 3}=\sqrt{2} K_{3} \bar{i}_{\alpha \beta \mathrm{II}} \\
& \bar{i}_{\alpha \beta 4}=\sqrt{2} K_{4} \bar{i}_{\alpha \beta \mathrm{II}}
\end{aligned}
$$

where $K_{1}, K_{2}, K_{3}$ and $K_{4}$ represent the current sharing coefficients of the four sub-winding sets. Note that sub-winding sets of the second motor are denoted with indices 3 and $4\left(\bar{l}_{\alpha \beta 3}\right.$ and $\left.\bar{\imath}_{\alpha \beta 4}\right)$. By substituting newly introduced current sharing coefficients of (31) into (30) the following relationships between the sub-motor $\alpha-\beta$ space vectors and sub-winding sets current space vectors can be obtained (note that the equations are given for the first sub-motor only, but the equivalent equations can be given for the second sub-motor):

$$
\begin{aligned}
& \bar{i}_{\alpha \beta \mathrm{I}}=\left(K_{1}+K_{2}\right) \bar{i}_{\alpha \beta \mathrm{I}} \\
& \bar{i}_{x y \mathrm{I}}=\left(K_{1}-K_{2}\right) \bar{i}_{\alpha \beta \mathrm{I}}^{*}
\end{aligned}
$$

From (32), the currents of the sub-winding sets can be controlled by changing the $K$ coefficients. These equations are valid in the common reference frame. Unlike $\bar{i}_{\alpha \beta}, \bar{i}_{x y}$ should be controlled in the anti-synchronous reference frame [2]. This will allow the control of the currents' amplitude of the subwinding sets in each sub-motor and consequently the power flow direction of each individual set. However, the transfer of power from one sub-motor to another is not possible by using the current sharing coefficients only. Thus, the sub-motors' power sharing coefficients $\left(G_{\mathrm{I}}\right.$ and $\left.G_{\mathrm{II}}\right)$ should be integrated with the current sharing coefficients of the sub-winding sets to obtain multidirectional power flow among the sub-winding sets of the double sub-motors.

As obvious from (32), the summation of each sub-motor's current sharing coefficients should always be equal to one $\left(K_{1}+K_{2}=1\right.$ and $K_{3}+K_{4}=1$, for the set II). Also, the sub-motor power sharing coefficients $\left(G_{\mathrm{I}}\right.$ and $\left.G_{\mathrm{II}}\right)$ must always sum to one, in order to maintain the same speed and torque. Fig. 4 illustrates the IRFOC schematic for the double-winding six-phase drive.

\section{SiMULATION RESULTS}

The proposed six-phase induction machine's control strategy is validated using Matlab/Simulink. The parameters of the simulated six-phase induction machine are presented in Table 1. The simulation results are illustrated in Fig. 5. The double six-phase machine is modelled and simulated as a twelve-phase machine in terms of phase variables considering all flux linkages between windings and sub-windings. This is done because such a machine cannot be modelled in terms of $d$ $q$ variables due to the $12 \times 12$ matrix not being invertible. The leakage flux (i.e. inductance) between two six-phase windings was taken as zero. The proposed IRFOC strategy shown in Fig. 4 is implemented. At first, the machine is started with $T_{l}=0$ until the machine settled at the reference speed of $500 \mathrm{rpm}$. Then, the machine was loaded with $4 \mathrm{Nm}$ at time instant of 1.8 sec. Through this period, the power sharing coefficients of the machine $G_{\mathrm{I}}$ and $G_{\mathrm{II}}$ are equal and both are 0.5 . Next, the power flow control within each sub-motor is validated, this means that the power transfer from the first to the second sub-winding set of each sub-motor is performed by changing current sharing coefficients of the sub-winding sets $\left(K_{1,2,3,4}\right)$ as shown in Fig. 5 from 2.0 to $2.5 \mathrm{sec}$. The current amplitude in each sub-winding can be seen to change proportionally with $K$. While the $d-q$ currents of the sub-motors are constant through the period 2.0 to $2.5 \mathrm{sec}$ the auxiliary $x-y$ currents can be seen to change accordingly.

To validate the power transfer ability from the first to the second sub-motor and vice versa, the power sharing coefficients between the sub-motors $G_{\mathrm{I}}$ and $G_{\mathrm{II}}$ were changed to 1.5 and -0.5 , respectively, during the period from 2.5 to $3.0 \mathrm{sec}$. During this period, the second sub-motor has changed its power flow direction at $2.5 \mathrm{sec}$. This can be noticed from the $q$-axis currents for the sub-motors $\left(i_{q \mathrm{I}}\right.$ and $\left.i_{q \mathrm{II}}\right)$ shown in Fig. 5. While the first sub-motor works in motoring mode, the other one is working in generation mode. This can be utilised in directing the power to a specific storage unit depending on it is state of charge (SOC).

Moreover, the proposed IRFOC algorithm is valid to control the double sub-motors machines during post-fault operating condition. It can be noticed that the proposed scheme maintained a constant speed and torque. However, an upper limit of the sub-winding sets' currents should be set, to ensure that the currents do not exceed the rated current of the winding insulation or the rated current of the power electronics conversion systems.

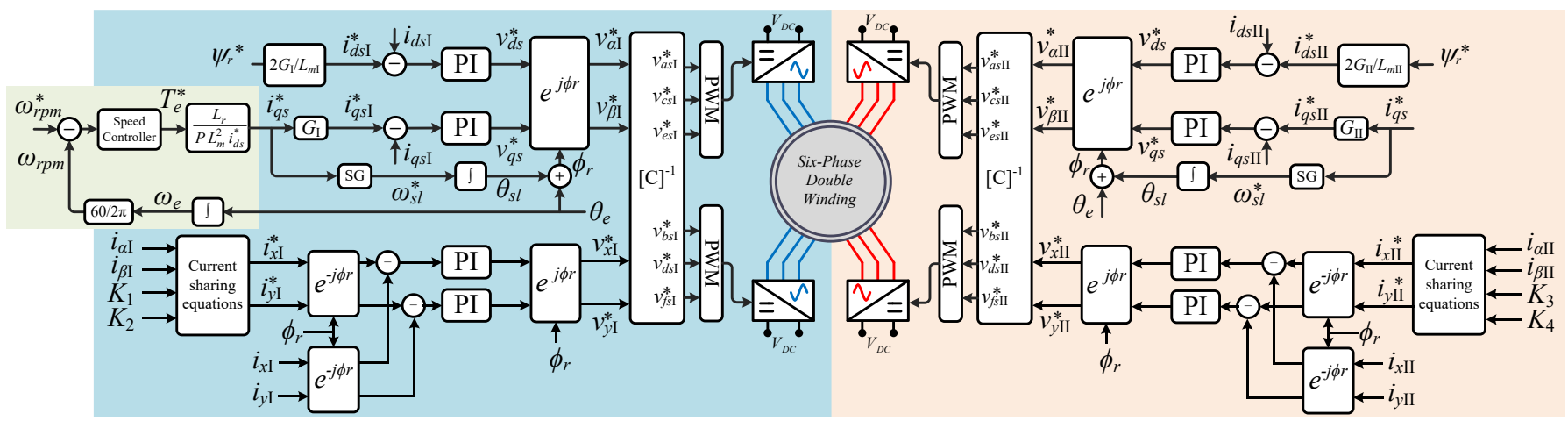

Fig. 4. IRFOC schematic for the double-winding six-phase induction machine. 
Table 1. Six-phase sub-motor parameters.

\begin{tabular}{|c|c|c|c|}
\hline$P_{\text {rated }}$ & $1.1 \mathrm{~kW}$ & $P$ & 3 (pair) \\
\hline$f_{\text {switching }}$ & $10 \mathrm{kHz}$ & $R_{r, \text { total }}$ & $1.8 \Omega$ \\
\hline$L_{l s \text { I II }}$ & $8.1 \mathrm{mH}$ & $R_{S I, \text { II }}$ & $3.6 \Omega$ \\
\hline$L_{l r, \text { total }}$ & $11.5 \mathrm{mH}$ & $M_{\mathrm{I}, \mathrm{II}}$ & $68.33 \mathrm{mH}$ \\
\hline$V_{D C}$ & $400 V_{D C}$ & $L_{m \text { I, II }}$ & $205 \mathrm{mH}$ \\
\hline
\end{tabular}
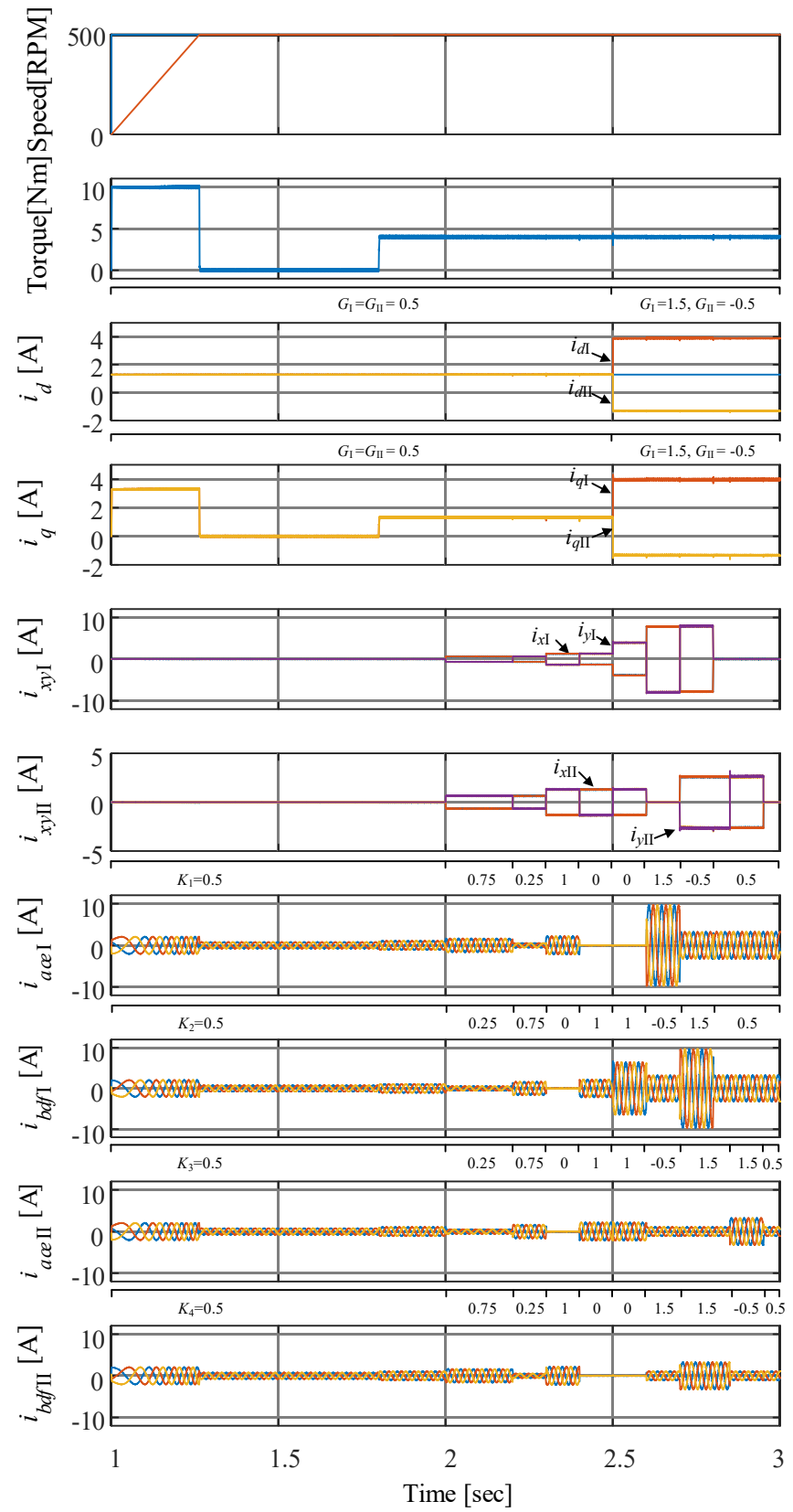

Fig. 5. Simulation results for the proposed IRFOC algorithm with multidirectional power flow.

\section{CONCLUSION}

In this paper, a novel IRFOC algorithm is developed for the six-phase induction machine with double layers of winding with the ability to control the power flow direction. The control scheme is utilising the auxiliary currents of the six-phase machine to control the power flow direction and amount of each sub-winding set. The proposed scheme is validated through Matlab/Simulink with different operating scenarios. The results illustrate the control scheme capability to control the power flow direction and amount within each sub-motor or from one sub-motor to another. Furthermore, the control scheme can be utilised to operate during the post-fault operation condition.

\section{ACKNOWLEDGMENT}

The authors would like to acknowledge the Engineering and Physical Sciences Research Council (EPSRC) for supporting the Power flow control in future electric vehicles and dc microgrids with multiple energy sources project (EP/P00914X/1).

\section{REFERENCES}

[1] H. S. Che, M. J. Duran, E. Levi, M. Jones, W. P. Hew, and N. A. Rahim, "Postfault operation of an asymmetrical six-phase induction machine with single and two isolated neutral points," IEEE Transactions on Power Electronics, vol. 29, pp. 5406-5416, 2014.

[2] H. S. Che, E. Levi, M. Jones, W. P. Hew, and N. A. Rahim, "Current control methods for an asymmetrical six-phase induction motor drive," IEEE Transactions on Power Electronics, vol. 29, pp. 407-417, 2014.

[3] M. Mengoni, G. Sala, L. Zarri, A. Tani, G. Serra, Y. Gritli, M. Duran, "Control of a fault-tolerant quadruple three-phase induction machine for more electric aircrafts," in Proc. IEEE Industrial Electronics Society Annual Conference, IECON, Florence, Italy, 2016, pp. 5747-5753.

[4] A. Tani, G. Serra, M. Mengoni, L. Zarri, G. Rini, and D. Casadei, "Dynamic stator current sharing in quadruple three-phase induction motor drives," in Proc. IEEE Industrial Electronics Society Annual Conference, IECON, Vienna, Austria, 2013, pp. 5173-5178.

[5] G. Grandi, A. Tani, P. Sanjeevikumar, and D. Ostojic, "Multi-phase multi-level AC motor drive based on four three-phase two-level inverters," in Proc. International Symposium on Power Electronics, Electrical Drives, Automation and Motion, SPEEDAM, Pisa, Italy, 2010, pp. 1768-1775.

[6] E. Levi, "Advances in converter control and innovative exploitation of additional degrees of freedom for multiphase machines," IEEE Transactions on Industrial Electronics, vol. 63, pp. 433-448, 2016.

[7] E. Levi, R. Bojoi, F. Profumo, H. A. Toliyat, and S. Williamson, "Multiphase induction motor drives - a technology status review," IET Electric Power Applications, vol. 1, pp. 489-516, 2007.

[8] I. Subotic, N. Bodo, E. Levi, M. Jones, and V. Levi, "Isolated chargers for EVs incorporating six-phase machines," IEEE Transactions on Industrial Electronics, vol. 63, pp. 653-664, 2016.

[9] I. Subotic, N. Bodo, E. Levi, B. Dumnic, D. Milicevic, and V. Katic, "Overview of fast on-board integrated battery chargers for electric vehicles based on multiphase machines and power electronics," IET Electric Power Applications, vol. 10, pp. 217-229, 2016.

[10] I. Subotic, N. Bodo, and E. Levi, "An EV drive-train with integrated fast charging capability," IEEE Transactions on Power Electronics, vol. 31, pp. 1461-1471, 2016.

[11] I. Subotic, N. Bodo, and E. Levi, "Single-phase on-board integrated battery chargers for EVs based on multiphase machines," IEEE Transactions on Power Electronics, vol. 31, pp. 6511-6523, 2016.

[12] G. Scarcella, G. Scelba, M. Cacciato, A. Spampinato, and M. M. Harbaugh, "Vector control strategy for multidirectional power flow in integrated multidrives starter-alternator applications," IEEE Transactions on Industry Applications, vol. 52, pp. 4816-4826, 2016.

[13] B. M. Wilamowski and J. D. Irwin, Power electronics and motor drives: CRC Press, 2011

[14] Y. Hu, Z. Q. Zhu, and K. Liu, "Current control for dual three-phase permanent magnet synchronous motors accounting for current unbalance and harmonics," IEEE Journal of Emerging and Selected Topics in Power Electronics, vol. 2, pp. 272-284, 2014

[15] Z. Liu, Z. Zheng, L. Xu, K. Wang, and Y. Li, "Current balance control for symmetrical multiphase inverters," IEEE Transactions on Power Electronics, vol. 31, pp. 4005-4012, 2016.

[16] H. S. Che, W. P. Hew, N. A. Rahim, E. Levi, M. Jones, and M. J. Duran, "Current control of a six-phase induction generator for wind energy plants," in Proc. Power Electronics and Motion Control Conference, EPE/PEMC, Novi Sad, Serbia, 2012, pp. LS5b.2-1-LS5b.2-7. 